\title{
Estratégias de cuidado para idosos com hiperplasia prostática benigna: uma revisão integrativa da literatura
}

\author{
Care strategies for the elderly with benign prostatic hyperplasia: an integrative literature review \\ Estrategias de atención para ancianos con hiperplasia benigna de próstata: revisión integradora de \\ la literatura
}

Dayara de Nazaré Rosa de Carvalho ORCID: https://orcid.org/0000-0001-8569-3392

Universidade Federal de São Carlos, Brasil

E-mail: dayara_twain@hotmail.com

Marcela Raíssa Asevedo Dergan

ORCID: https://orcid.org/0000-0003-1457-0242 Universidade do Estado do Pará, Brasi

E-mail: derganm20@gmail.com

Paula Andreza Viana Lima

ORCID: https://orcid.org/0000-0002-8217-8288

Universidade Federal do Amazonas, Brasil E-mail: paulaviana_lima@hotmail.com

Tainan Fabrício da Silva

ORCID: https://orcid.org/0000-0001-6761-1365

Universidade Federal do Amazonas, Brasil E-mail: tainanfabricio@hotmail.com

Lucas de Jesus Pereira

ORCID: https://orcid.org/0000-0002-3938-3286 Universidade de São Paulo, Brasil

E-mail: lucas-laje@hotmail.com

Natalie Kesle Costa Tavares

ORCID: https://orcid.org/0000-0002-0482-6999

Universidade Federal do Amazonas, Brasil

E-mail: natalietavares.nt@gmail.com

Layana Giselly Silva Ferreira

ORCID: https: //orcid.org/0000-0002-6471-9511

Universidade Federal de São Carlos, Brasil.

E-mail: layanagiselly@gmail.com

Danielle Maria Martins Carneiro

ORCID: https://orcid.org/0000-0002-1787-4332

Universidade do Estado do Pará, Brasil

E-mail: danielle.carneiro@ uepa.com

Nathila Keith Câmara Borges

ORCID: https://orcid.org/0000-0002-4300-8294 Hospital Universitário João de Barros Barreto, Brasil

Email:nathilaborges@yahoo.com.br

Camila Medeiros Maciel

ORCID: https://orcid.org/0000-0002-8901-2125

Faculdade Integrada da Amazônia, Brasil.

E-mail: camilammaciel1@gmail.com

Fernanda de Nazaré Almeida Costa

ORCID: https: //orcid.org/0000-0002-0544-4378

Universidade Federal do Rio de Janeiro, Brasil

E-mail: fepedrinho@yahoo.com.br

Daniele Moura de Souza

ORCID: https://orcid.org/0000-0001-6479-0580 Hospital Universitário João de Barros Barreto, Brasil

E-mail: danimoura2001@ yahoo.com.br

Ana Carolina Martins Damaceno

ORCID: https://orcid.org/0000-0003-1328-8050 Escola Superior da Amazônia, Brasil

E-mail: carol_martins_damaceno@hotmail.com

Miriam Souza Oliveira

Centro Universitário Metropolitano da Amazônia, Brasil ORCID: https://orcid.org/0000-0002-0561-631X

E-mail: miriamsouzaoliveira123@gmail.com 
Charles Carvalho dos Santos

ORCID: https://orcid.org/0000-0002-8572-0482

Universidade da Amazônia, Brasil

E-mail: charlestcheik@hotmail.com

Larissa Graziela Sousa da Silva

ORCID: https://orcid.org/0000-0002-5853-334X

Hospital universitário Getúlio Vargas, Brasil

E-mail: laragazi@gmail.com

Monique Lindsy de Souza Baia

ORCID: https://orcid.org/0000-0002-5660-5223

Universidade do Estado do Pará, Brasi

E-mail: moniquelindsy@ hotmail.com

Yves de Luka Miranda dos Santos

ORCID: https://orcid.org/0000-0002-1244-6056

Unifamaz, Brasil

E-mail: lukamiranda0@gmail.com

Dayanne de Nazare dos Santos

ORCID: https://orcid.org/0000-0002-6389-7287

Universidade do Estado do Pará, Brasil

E-mail: enfdayannesantos@yahoo.ckm.br

Tamires da Silva Dutra

ORCID: https://orcid.org/0000-0002-4916-6672

Pontifícia Universidade Católica do Paraná, Brasil.

E-mail: tammy.sd@hotmail.com

Fabiana de Souza Orlandi

ORCID: https://orcid.org/0000-0002-5714-6890

Universidade Federal de São Carlos, Brasil

E-mail: forlandi@ufscar.br

\begin{abstract}
Resumo
Objetivo: Demonstrar através de uma Revisão Interativa de Literatura, as estratégias de cuidado ao paciente idoso com Hiperplasia Benigna Prostática (HPB). Método: Trata-se de um estudo de Revisão Integrativa da Literatura (RIL), com a realização da coleta de dados por meio de acesso on-line nas bases e bancos de dados Cochrane Library; PubMed, Cochrane Library e BVS. Após avaliação e sintetização dos artigos, os dados foram analisados por meio do software IraMuTeQ, apresentando os resultados por meio da análise de similitude com a identificação das coocorrências entre as palavras e indicações da conexidade. Resultados e Discussão: A partir dos resultados obtidos através da árvore de coocorrência, foram divididas 3 classes: A prevalência da relação entre HPB e LUTS como forma de detecção de agravos; Avaliação da população idosa com HPB como forma de identificar limitação, causa ou condição que reduza a eficácia e efeito do tratamento. As intervenções dos sintomas de HPB em idosos. Conclusão: Observa-se que as estratégias de cuidado para idosos com Hiperplasia Benigna Prostática ainda são escassas, limitando-se as intervenções farmacológicas e comportamentais. A avaliação das especificidades dessa população apresenta-se como uma forma de monitorar os resultados provenientes dos tratamentos, além dos efeitos adversos afins de evitar o agravamento do quadro.
\end{abstract}

Palavras-chave: Idoso; Hiperplasia Prostática Benigna; LUTS.

\begin{abstract}
Objective: Demonstrate, through an Interactive Literature Review, care strategies for elderly patients with Benign Prostatic Hyperplasia (BPH). Methods: This is an Integrative Literature Review (RIL) study, with data collection through online access in Cochrane Library databases and databases; PubMed, Cochrane Library and VHL. After evaluating and synthesizing the articles, the data were analyzed using the IraMuTeQ software, presenting the results through the similarity analysis with the identification of co-occurrences between the words and indications of the connection. Results/Discussion: From the results obtained through the co-occurrence tree, 3 classes were divided: The prevalence of the relationship between HBP and LUTS as a way of detecting diseases; Evaluation of the elderly population with BPH as a way to identify limitation, cause or condition that reduces the effectiveness and effect of the treatment.; Interventions for BPH symptoms in the elderly. Conclusion: It is observed that care strategies for elderly people with Benign Prostatic Hyperplasia are still scarce, limiting pharmacological and behavioral interventions. The assessment of the specificities of this population is presented as a way to monitor the results from the treatments, in addition to adverse effects related to avoiding the worsening of the condition.
\end{abstract}

Keywords: Elderly; Benign prostatic hyperplasia; LUTS.

\title{
Resumen
}

Objetivo: Demostrar, a través de una Revisión Interactiva de la Literatura, las estrategias de atención al paciente anciano con Hiperplasia Benigna de Próstata (HPB). Método: Se trata de un estudio de revisión de literatura integradora (RIL), con la recopilación de datos realizada a través del acceso en línea en las bases de datos y bases de datos de la Biblioteca Cochrane; PubMed, Cochrane Library y VHL. Luego de evaluar y sintetizar los artículos, los 
datos fueron analizados utilizando el software IraMuTeQ, presentando los resultados mediante el análisis de similitud con la identificación de co-ocurrencias entre las palabras y las indicaciones de la conexión. Resultados y Discusión: Con base en los resultados obtenidos a través del árbol de co-ocurrencia, se dividieron 3 clases: La prevalencia de la relación entre HPB y STUI como una forma de detección de enfermedades; Evaluación de la población anciana con HPB como forma de identificar limitación, causa o condición que reduce la efectividad y efecto del tratamiento. Intervenciones para los síntomas de HPB en ancianos. Conclusión: Se observa que las estrategias de atención a las personas mayores con Hiperplasia Benigna de Próstata aún son escasas, limitando las intervenciones farmacológicas y conductuales. La valoración de las especificidades de esta población se presenta como una forma de monitorizar los resultados de los tratamientos, además de los efectos adversos relacionados con evitar el agravamiento de la afección.

Palabras clave: Anciano; Hiperplasia prostática benigna; LUTS.

\section{Introdução}

A Hiperplasia Prostática Benigna (HPB) é caracterizada pelo aumento progressivo da próstata a partir de alterações na proliferação das células epiteliais da musculatura lisa do órgão (Bortnick et al., 2019). É uma doença comum em homens idosos e está crescendo o número de casos à medida que a população global envelhece (Shore, 2015). Manifestações clínicas da hiperplasia prostática são micção frequente, urgência, incontinência, infecção do trato urinário, comprometimento da função renal e inflamação da próstata (Zhang et al, 2015).

Considerando o aspecto histológico, inicia-se com uma hiperplasia microtubular simples, das células do estroma e do epitélio da glândula prostática, com uma consequente expansão nodular macroscópica, gerando o aumento benigno da próstata e numa possível interferência no fluxo normal da urina, ocasionada pela compressão da uretra e pelo relaxamento inadequado do colo vesical, podendo levar a Sintomas do Trato Urinário Inferior (LUTS) (Rył et al, 2015)

A HPB causa disfunção urinária em homens de meia idade e idosos, com efeitos adversos na vida dos pacientes. O envelhecimento e a função testicular anormal têm sido considerados as principais causas da HPB. Com o aumento da idade, bem como os níveis de estrogênio, testosterona e di-hidrotestosterona, o corpo perde o equilíbrio. Isso pode resultar em distúrbios dos hormônios sexuais em pacientes do sexo masculino, levando à hipertrofia da próstata, estreitamento do orifício uretral. Isso leva ainda à obstrução uretral e à disfunção miccional, que afetam seriamente o esvaziamento da bexiga e da urina (Dai et al, 2016. Lokant \& Naz, 2015)

O aumento do volume da próstata na velhice varia entre 2,0\% e 2,5\% ao ano, e a prevalência é superior a 70\% entre os homens acima de 60 anos (Alawamlh et al, 2018). Enquanto a HPB raramente causa problemas antes dos 40 anos, os sintomas afetam a vida diária e a qualidade de vida dos indivíduos idosos. (Cureklibatir \& Kizilay, 2015).

A HPB e seus sintomas causam um impacto de caráter negativo a nível emocional, social e econômico do paciente, assim na vida de seus familiares, amigos e cuidadores tornando-se num grande desafio aos profissionais de saúde na busca de alternativas, abordagens e medidas terapêuticas (Teixeira et al., 2017).

A atenção em saúde para a hiperplasia prostática benigna apresenta extrema importância, já que esta patologia se não tratada adequadamente, pode apresentar complicações como infecção urinária, litíase vesical, e até deterioração do trato urinário superior (rins) em casos mais extremos, levando ao comprometimento da qualidade vida do idoso e até ao maior risco de óbito (Barbosa \& Antunes, 2018).

Diante disto, este estudo tem como objetivo demonstrar através de uma Revisão Interativa de Literatura nacional e internacional, as estratégias de cuidado ao paciente idoso com Hiperplasia Benigna Prostática (HPB) entre os anos de 2014 a 2019. 


\section{Método}

Trata-se de um estudo de Revisão Integrativa da Literatura (RIL), realizada em seis etapas distintas apresentadas a seguir: 1) Identificação do tema, problema de pesquisa e objeto de estudo; 2) Estabelecimento de critérios de busca nas principais bases de dados; 3) Categorização dos estudos e coleta de dados por meio de instrumento; 4) Avaliação dos estudos e coleta de dados; 5) Apresentação dos resultados; 6) Discussão e apresentação da Revisão Integrativa da Literatura.

A partir do objeto de estudo, elaborou-se a seguinte questão de pesquisa: Quais as estratégias de cuidados prestada ao idoso com hiperplasia prostática benigna disponíveis na literatura nacional e internacional?

A coleta de dados ocorreu por meio de acesso on-line nas seguintes bases e bancos de dados: Cochrane Library; PubMed, Cochrane Library e BVS. Para otimizar e refinar a busca e garantir o direcionamento para todos os trabalhos pertinentes a temática, a seleção dos artigos ocorreu a partir da combinação de descritores controlados cadastrados no DeCS (Descritores em Ciências da Saúde) como: “idoso"; "estratégias de cuidado"; "hiperplasia prostática" e "saúde do homem" mediados pelo operador booleano "AND", visando ampliar o quantitativo de estudos. Foram ainda utilizados os descritores não controlados "cuidado a hiperplasia prostática" e "intervenções para hiperplasia prostática".

Os critérios de inclusão estabelecidos para a elaboração desta RIL foram: artigos de pesquisa do tipo pesquisa de campo e Revisão Sistemática, publicados no período de 2010 a 2019, nos idiomas português, inglês e espanhol, disponíveis eletronicamente e na íntegra e que abordavam sobre a temática. Foram excluídos estudos do tipo: editoriais, cartas ao editor, artigos incompletos, estudos reflexivos, literatura cinza e estudos que não abordavam temática relevante ao objetivo do estudo.

Ao final da coleta de dados emergiram um total de 34 artigos nas referidas bases e banco de dados. Após essa etapa, os artigos passaram por avaliação quanto ao título e resumo e deste total, 27 foram excluídos do processo de triagem. A avaliação durante essa etapa objetivou descartar artigos irrelevantes. Ao final da coleta de dados emergiram 7 artigos que foram avaliados quanto ao título do artigo, autores, ano e base de dados, tipo de estudo e publicação, objetivo e síntese dos resultados e que respondiam a questão de pesquisa.

Ao final desta etapa, emergiu a amostragem final, composta por 7 publicações, os quais atendiam aos critérios estabelecidos, conforme apresenta o Quadro 1 a seguir:

Quadro 1 - Identificação dos artigos selecionados.

\begin{tabular}{|c|c|c|c|c|}
\hline $\begin{array}{l}\text { TÍTULO DO } \\
\text { ARTIGO }\end{array}$ & $\begin{array}{c}\text { Autores / } \\
\text { Ano / Base } \\
\text { de Dados }\end{array}$ & $\begin{array}{c}\text { Tipo de } \\
\text { Estudo / País } \\
\text { de } \\
\text { Publicação } \\
\end{array}$ & Objetivo & Síntese dos Resultados \\
\hline $\begin{array}{l}\text { 1-Moxibustion as an } \\
\text { adjunct for lower urinary } \\
\text { tract } \\
\text { symptoms associated } \\
\text { with benign prostate } \\
\text { enlargement }\end{array}$ & $\begin{array}{l}\text { Lee et al. } \\
2019 . \\
\text { PubMed }\end{array}$ & $\begin{array}{l}\text { Caso } \\
\text { controle. } \\
\text { Coreia do } \\
\text { Norte }\end{array}$ & $\begin{array}{l}\text { Analise a eficácia e segurança de } \\
\text { moxabustão adicional em } \\
\text { combinação com tratamento } \\
\text { convencional em comparação com } \\
\text { o tratamento convencional. }\end{array}$ & $\begin{array}{l}\text { O tratamento com moxabustão pode ser uma terapia adjuvante } \\
\text { benéfica para LUTS em pacientes com aumento benigno da } \\
\text { próstata. Um estudo de tamanho real com modificações de } \\
\text { comparador como tratamento simulado e um período de estudo } \\
\text { estendido é garantido para confirmar a eficácia desta terapia } \\
\text { adjuvante. Além disso, seria melhor se um número suficiente de } \\
\text { participantes em subgrupos fosse incluído para identificar os } \\
\text { efeitos em pacientes com sintomas atípicos ou aqueles que não } \\
\text { respondem bem ao tratamento convencional. }\end{array}$ \\
\hline $\begin{array}{l}\text { 2-Impact of metabolic } \\
\text { syndrome-related factors } \\
\text { on the development of } \\
\text { benign prostatic } \\
\text { hyperplasia and lower } \\
\text { urinary tract symptoms } \\
\text { in Asian population }\end{array}$ & $\begin{array}{c}\text { Park et al. } \\
2019 . \\
\text { PubMed. }\end{array}$ & $\begin{array}{c}\text { Estudo } \\
\text { retrospectivo } \\
\text { Coreia do Sul }\end{array}$ & $\begin{array}{l}\text { Avaliar se os fatores de risco para } \\
\text { síndromes metabólicas, incluindo } \\
\text { variáveis de estilo de vida, estão } \\
\text { relacionadas a os preditores de HPB } \\
\text { / LUTS no diagnóstico inicial e para } \\
\text { o desenvolvimento de HPB / LUTS } \\
\text { dentro de um acompanhamento de } 5 \\
\text { anos em um programa de saúde de } \\
\text { grande escala. }\end{array}$ & $\begin{array}{l}\text { A LUTS é comum entre homens idosos; no entanto, pode estar } \\
\text { relacionado a doenças malignas da próstata. Um grande estudo } \\
\text { europeu recente de base populacional estabeleceu uma } \\
\text { associação clara entre STUI e o risco subsequente de câncer de } \\
\text { próstata. Em conclusão, as variáveis relacionadas à síndrome } \\
\text { metabólica, incluindo massa gorda, a polipoproteína B, glicemia } \\
\text { de jejum, colesterol, HDL e níveis de LDL, estão } \\
\text { significativamente associadas ao desenvolvimento da LUTS, e o } \\
\text { aumento da massa gorda e dos níveis de LDL parecem ser } \\
\text { importantes para impedindo o desenvolvimento de LUTS. Mais } \\
\text { estudos investigando a intercorrelação entre cada componente da } \\
\text { síndrome metabólica são necessários para validar os resultados } \\
\text { do presente estudo. }\end{array}$ \\
\hline $\begin{array}{l}\text { 3-Metformin reduces } \\
\text { prostate cancer risk }\end{array}$ & $\begin{array}{l}\text { Kuo et al. } \\
2019 .\end{array}$ & $\begin{array}{l}\text { Estudo de } \\
\text { coorte }\end{array}$ & $\begin{array}{l}\text { Avaliar a eficácia da metformina } \\
\text { para diabéticos com diagnóstico de }\end{array}$ & $\begin{array}{l}\text { Nossos dados mostram que o medicamento metformina pode } \\
\text { reduzir o risco de câncer de próstata em } 40 \% \text { dos pacientes }\end{array}$ \\
\hline
\end{tabular}




\begin{tabular}{|c|c|c|c|c|}
\hline $\begin{array}{l}\text { among men with benign } \\
\text { prostatic hyperplasia: A } \\
\text { nationwide } \\
\text { population-based cohort } \\
\text { study }\end{array}$ & PubMed. & $\begin{array}{l}\text { retrospectivo. } \\
\text { Taiwan }\end{array}$ & $\begin{array}{l}\text { HPB. Pacientes com HPB com e } \\
\text { sem terapia com metformina foram } \\
\text { comparados a ocorrência de câncer } \\
\text { de próstata durante o período de } \\
\text { acompanhamento. }\end{array}$ & $\begin{array}{l}\text { diabéticos com HPB. Homens limitados com HPB são usuários } \\
\text { de metformina. Aqueles que usam a medicina chinesa também se } \\
\text { beneficiaram com o tratamento. É razoável supor que o início } \\
\text { precoce do tratamento com metformina pode reduzir ainda mais } \\
\text { o risco de câncer de próstata. Estudos futuros para avaliar a } \\
\text { dosagem mais adequada de metformina para pacientes com HPB } \\
\text { são essenciais. }\end{array}$ \\
\hline $\begin{array}{l}\text { 4-Hyperlipidemia is } \\
\text { associated with an } \\
\text { increased risk of clinical } \\
\text { benign prostatic } \\
\text { hyperplasia }\end{array}$ & $\begin{array}{l}\text { Shih et al. } \\
2017 . \\
\text { PubMed. }\end{array}$ & $\begin{array}{l}\text { Estudo de } \\
\text { coorte } \\
\text { longitudinal. } \\
\text { Taiwan }\end{array}$ & $\begin{array}{l}\text { Analise se a hiperlipidemia está } \\
\text { associada a um risco aumentado de } \\
\text { HPB. }\end{array}$ & $\begin{array}{l}\text { Pacientes com hiperlipidemia estão associados a um risco } \\
\text { aumentado de desenvolver HPB em comparação com pacientes } \\
\text { sem hiperlipidemia. }\end{array}$ \\
\hline $\begin{array}{l}\text { 5- The association of } \\
\text { endothelial nitric oxide } \\
\text { synthase (eNOS) G894T } \\
\text { gene polymorphism with } \\
\text { responsiveness to a } \\
\text { selective a1-blocker in } \\
\text { men with benign } \\
\text { prostatic hyperplasia } \\
\text { related lower urinary } \\
\text { tract symptoms }\end{array}$ & $\begin{array}{l}\text { Lee et al. } \\
2016 . \\
\text { PubMed. }\end{array}$ & $\begin{array}{l}\text { Estudo } \\
\text { transversal. } \\
\text { EUA. }\end{array}$ & $\begin{array}{l}\text { Investigar prospectivamente uma } \\
\text { associação de endoteliais } \\
\text { polimorfismo do gene G894T da } \\
\text { óxido nítrico sintase (eNOS) } \\
\text { resposta a um bloqueador a1 } \\
\text { selecionado em homens com } \\
\text { hiperplasia benigna da próstata com } \\
\text { sintomas do trato urinário inferior } \\
\text { (HPB / LUTS). }\end{array}$ & $\begin{array}{l}\text { A presença do alelo eNOS 894T teve um impacto } \\
\text { significativamente negativo na resposta a um bloqueador } \alpha 1 \\
\text { seletivo no tratamento de Hiperplasia Prostática Benigna / } \\
\text { LUTS, sugerindo que o polimorfismo do gene eNOS G894T } \\
\text { pode ser um fator de suscetibilidade genética para a eficácia do } \\
\text { bloqueador } \alpha 1 \text { em homens com HPB / LUTS. }\end{array}$ \\
\hline
\end{tabular}

Fonte: Autores da pesquisa (2021).

Após avaliação e sintetização dos artigos, os dados foram analisados por meio do software IRaMuTeQ (Interface de $\mathrm{R}$ pour lês Analyses Multidimensionnelles de Textes et de Questionnaires), que foi desenvolvido na França por Pierre Ratinaud (2009). O programa se ancora no software R e permite diferentes formas de análises estatísticas sobre o corpus textuais e tabelas de indivíduos por palavras.

O corpus textual foi construído a partir dos resultados e conclusão dos estudos incluídos e colocados em um único arquivo de texto onde foram feitas leituras, correções e codificações. Para realizar análises lexicais clássicas, o software identifica e reformata as unidades de texto, que se transformam de Unidades de Contextos Iniciais (UCI) em Unidades de Contexto Elementar (UCE).

O IRaMuTeQ viabiliza 5 tipos de análises textuais e para este estudo foi utilizado a análise de similitude. Para Marchand e Ratinaud (2012), esse tipo de análise baseia-se na teoria dos grafos e possibilita identificar as coocorrências entre 
as palavras e seu resultado traz indicações da conexidade entre as palavras, auxiliando na identificação da estrutura da representação.

\section{Resultados e Discussão}

Conforme é observado na Figura 1, a árvore é apresentada na interface dos resultados da análise de similitude com a identificação das coocorrências entre as palavras e indicações da conexidade entre os termos: BPH (hiperplasia prostática benigna), LUTS (lower urinary tract symptom), patiente, man, prostate e treatment, auxiliando desta forma na identificação da estrutura do campo representacional dos fatores associados a hiperplasia prostática.

Figura 1: Análise de similitude.

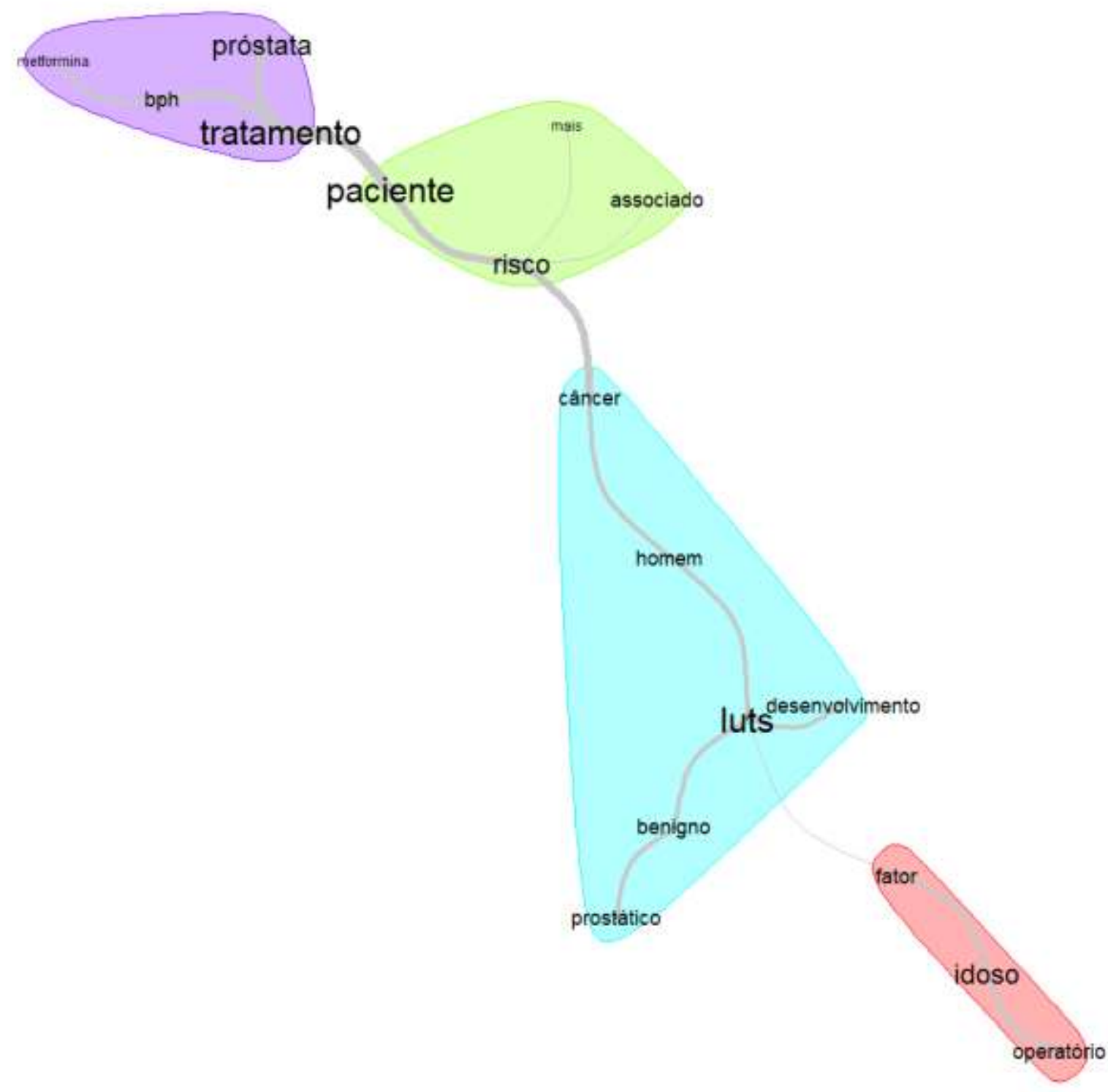

Fonte: Autores da pesquisa (2021). 
A partir dos resultados obtidos através da árvore de coocorrência, foram divididas 3 classes: A prevalência da relação entre HPB e LUTS como forma de detecção de agravos; Avaliação da população idosa com HPB como forma de identificar limitação, causa ou condição que reduza a eficácia e efeito do tratamento; As intervenções dos sintomas de HPB em idosos.

\section{Classe 1: A prevalência da relação entre HPB e LUTS como forma de detecção de agravos}

A prevalência de HPB com LUTS foi relatada entre 50\% e 75\% em homens com mais de 50 anos. De acordo com Lee et al (2019), a BPH é a causa mais comum de LUTS no envelhecimento masculino, que pode afetar consideravelmente a qualidade de vida dos pacientes. Cerca de 50\% dos homens com HPB histologicamente confirmada têm LUTS, cujos sintomas incluem fluxo urinário fraco, sensação de esvaziamento incompleto da bexiga, hesitação urinária, micção frequente e incontinência de urgência

É considerado um agravo na saúde do homem por interferir nas atividades diárias e também alterar os padrões normais do sono, já que a patologia tem como principal sintoma o aumento da micção noturna (Lee et al, 2019).

Os dados epidemiológicos mostram uma prevalência crescente de HPB/LUTS à medida que os homens envelhecem, com 26\% com 40-50 anos de idade afetados aumentando para taxas de até 79\% em homens com idade $>70$ anos (Dai et al. 2016).

O estudo realizado por Lee et al (2016), analisou que a HPB pode causar LUTS parcialmente através do aumento da estimulação adrenérgica $\alpha$, levando a um aumento do tônus muscular liso uretral. Os bloqueadores selectivos $\alpha 1$ são bem conhecidos por serem uma opção de tratamento eficaz e não-invasivo para homens com BPH/LUTS, sendo considerados a primeira linha de tratamento farmacológico padrão. Park et al (2019) observou a existência de vários fatores de risco para o desenvolvimento e progressão da HPB e LUTS e, curiosamente, além do volume da próstata e antígeno prostático específico, as síndromes metabólicas foram consideradas determinantes importantes no desenvolvimento e na progressão do LUTS.

As diversas variáveis, incluindo componentes da síndrome metabólica, como apolipoproteína B, glicemia de jejum, colesterol, HDL (high density lipoprotein) e níveis de LDL (low density lipoprotein), foram significativamente associadas à HPB e LUTS no diagnóstico inicial. Entre as variáveis da síndrome metabólica, a diminuição da massa gorda e dos níveis de LDL foram fatores preditivos para o desenvolvimento de HPB e LUTS. (Park, 2019).

KUO (2019) indicou em seu estudo que as evidências epidemiológicas e genéticas têm uma forte ligação entre a HPB e o câncer de próstata e que o uso de metformina foi associado a uma diminuição do risco de câncer de próstata em homens com HPB. Logo, a incorporação de HPB e LUTS como parâmetro para prever o câncer de próstata seria interessante e poderia ajudar a aumentar a precisão da previsão do risco de câncer de próstata.

\section{Classe 2: Avaliação da população idosa com HPB como forma de identificar limitação, causa ou condição que reduza a eficácia e efeito do tratamento}

Nota-se a necessidade de conhecer e avaliar aspectos dos idosos com HPB tais como a obesidade, consumo de tabaco, sedentarismo, assim como o consumo alimentar e reação aos tratamentos propostos como forma de monitorar os resultados do tratamento e evitar agravos que afetam significativamente a sua qualidade de vida, visto que essa população apresenta especificidades que podem acarretar a resultados distintos (Barbosa \& Antunes, 2018).

Estudo realizado por Shih et al (2018) mostra que a obesidade pode aumentar os riscos de agravos do HPB, devido ao aumento dos níveis séricos de estrógenos e por piorar os sintomas do sistema urinários inferior, pelo aumento da atividade do sistema nervoso simpático. Além disso, considera-se que a síndrome metabólica (SM), em associação com diabetes tipo II, hipertensão, obesidade e dislipidemia, estariam envolvidas com o aparecimento ou agravamento do HPB. 
Os hábitos saudáveis são fatores protetores que devem ser incentivados para a eficácia do tratamento. Um estudo de meta-análise sugere que a atividade física moderada a vigorosa reduz o risco de agravos de HPB em $25 \%$ relativamente a um estilo de vida sedentário (Parsons et al., 2013).

A baixa adesão a dieta é um fator que precisa ser avaliado, visto que a quantidade calórica ingerida e uma série de alimentos tais como, as carnes vermelhas, a gordura, o leite e derivados aumentam potencialmente o risco de agravos de HPB ao passo que vegetais, frutas ácidos gordos polinsaturados, ácido linoleico vitamina A e vitamina D estão associados a um decréscimo do risco de HPB e LUTS (Kuo et al. 2019).

Park et al (2019) apresentou a associação de forma inversa entre a presença de licopeno, selénio e caroteno com HPB e LUTS. Além disso, o uso do zinco e da vitamina C tanto têm sido associados ao decréscimo do HPB e a melhor qualidade de vida dos pacientes.

Para Bitencourt et al. (2016), a monitorização dos profissionais afim de identificar aspectos que retardem ou afetem a eficácia do tratamento de idosos com eliminação urinária prejudicada mostra-se como uma forma de promover a qualidade do tratamento, além de observar os resultados apresentados pelas medicações, efeitos colaterais e especificidades desses pacientes.

\section{Classe 3: As intervenções dos sintomas de HPB em idosos}

Existem diferentes formas de intervenções para a HPB, incluindo a terapia não invasiva e terapias cirúrgicas. O tratamento conservador, incluindo espera vigilante e modificação de comportamento e dieta, é recomendado como um primeiro passo para pacientes com sintomas leves ou moderados, e a intervenção farmacológico é recomendado como um passo seguinte (Wroclawski et al., 2014).

Park et al (2019), evidencia a importância das modificações comportamentais como manter um estilo de vida saudável, parar de fumar e consumir álcool, aumentar os exercícios que levam à normalização da glicose no sangue, níveis de colesterol, etc., e eventualmente diminuem as síndromes metabólicas podendo aliviar os sintomas da doença.

Os tratamentos farmacológicos são determinados pelo tamanho da próstata, nível de antígeno prostático específico (PSA), sintomas acompanhantes ou fatores de risco. Os bloqueadores $\alpha$ são geralmente considerados como a primeira opção de tratamento devido à boa eficácia e ao baixo risco de eventos adversos graves (EA), mas não podem impedir a progressão (isto é, retenção urinária ou condições que exijam operação) e podem induzir disfunção ejaculatória (Santos et al. 2018).

Bortnick (2019) destaca em seu estudo métodos eficazes como a ressecção transuretral da próstata (também conhecido como RTUP ou RTU de próstata) que é um procedimento cirúrgico padrão para pacientes com HPB associada à LUTS, assim como as terapias alternativas incluindo MTOPS (Terapia Médica dos Sintomas da Próstata) e CombAT (Combinação de Avodart e Tamsulosin), modtrando-se bem tolerados e com poucos efeitos adverso.

Já o estudo realizado por Lee et al (2020), aborda sobre a moxabustão, que é um método de tratamento semelhante à acupuntura e envolve a estimulação dos pontos de acupuntura usando calor, como forma de terapia adjuvante para melhorar a LUTS em pacientes com HPB. Concluiu-se que a moxabustão apresenta eficácia no alívio de sintomas do aumento da próstata, especialmente esvaziamento incompleto, esforço, intermitência, noctúria e qualidade de vida em geral.

Bitencourt, Felippe, Santana (2016), destacam em seu estudo os principais Diagnósticos de Enfermagem para idosos com eliminação urinária prejudicada, evidenciando o modelo metodológico da Sistematização da Assistência de Enfermagem (SAE) como um importante e indispensável instrumento de trabalho não só do enfermeiro como também de toda a equipe, por subsidiar o processo de identificação das necessidades de cuidados e otimizar a organização e o planejamento das ações a serem executados.

Evidencia-se a importância da assistência à saúde do homem idoso desempenhado pelo profissional de enfermagem, sabendo que é um processo amplo e complexo, pois envolve medidas promocionais da saúde, preventivas, terapêuticas e, 
principalmente, de interação com o cliente. E no meio hospitalar, o enfermeiro, além de responsável pela assistência prestada aos pacientes, também recebe a responsabilidade de exercer a liderança da equipe de enfermagem e principalmente pela organização e planejamento, entre os cuidados prestados, que se concretizam na implantação da Sistematização da Assistência de Enfermagem (Farias \& Castillo, 2020).

\section{Conclusão}

Observa-se que as estratégias de cuidado para idosos com Hiperplasia Benigna Prostática ainda são escassas, limitando-se as intervenções farmacológicas e comportamentais. A avaliação das especificidades dessa população apresenta-se como uma forma de monitorar os resultados provenientes dos tratamentos, além dos efeitos adversos afins de evitar o agravamento do quadro.

Destaca-se a necessidade da produção de mais conteúdo cientifico referente à temática dada à escassez de artigos abordando a assistência ao idoso com BHP e considerando a sua importância e prevalência, entretanto é evidente a importância da figura do enfermeiro tanto na prevenção quanto no tratamento dessa condição devido a sua competência e por ser um dos profissionais que possuem maior contado com os pacientes.

\section{Referências}

Alawamlh, O. A. H., Goueli, R., \& Lee, R. K. (2018). Lower Urinary Tract Symptoms, Benign Prostatic Hyperplasia, and Urinary Retention. Med Clin North Am; 102(2): 301-11.https://www.ncbi.nlm.nih.gov/pubmed/29406059

Bitencourt, G. R., Felippe, N. H. M. D., \& Santana, R. F. (2016). Diagnóstico de enfermagem eliminação urinária prejudicada em idosos no pós-operatório: um estudo transversal. Rev enferm UERJ, 24(3). https://www.e-publicacoes.uerj.br/index.php/enfermagemuerj/article/view/16629.

Barbosa, J. A., \& Antunes, A. (2018). Técnicas minimamente invasivas para o tratamento da hiperplasia prostática benigna. Revista De Medicina, 97(3), 314319. https://doi.org/10.11606/issn.1679-9836.v97i3p314-319

Bortnick, E. M., Simma-chiang, V., \& Kaplan, A. S. (2019). Long-term Consequences of Medical Therapy for Benign Prostatic Hyperplasia. Reviews in Urology 21(4). https://www.ncbi.nlm.nih.gov/pmc/articles/PMC7020283/.

Cureklibatir, I., \& Kizilay, F. (2015). Prostate Gland and its Diseases. Ege University Public Books Series; 18.

Dai, X. et al. (2016). "Benign Prostatic Hyperplasia and the Risk of Prostate Cancer and Bladder Cancer: A Meta-Analysis of Observational Studies." Medicine 95(18). https://www.ncbi.nlm.nih.gov/pmc/articles/PMC4863764/

Farias, T. L. F., \& Castillo, L. A. C. (2020). Assistência de enfermagem ao portador de câncer de próstata noserviço de urgência/emergência: uma revisão bibliográfica. Revista Científica Educandi \& Civitas, 3(1) http://162.241.180.121/ educandiecivitas/index.php/educandiecivitas/article/view/38.

Kuo, Y. J. et al. (2019). Metformin reduces prostate cancer risk among men with benign prostatic hyperplasia: A nationwide population-based cohort study. Cancer Medicine. 1-10. https://www.ncbi.nlm.nih.gov/pmc/articles/PMC6536940/.

Lee, Y. C. et al. (2016). The association of endothelial nitric oxide synthase (eNOS) G894T gene polymorphism with responsiveness to a selective a1-blocker in men with benign prostatic hyperplasia related lower urinary tract symptoms. BJU Int. 118(2):313-9. https://www.ncbi.nlm.nih.gov/pubmed/26940040.

Lee, H. Y. et al. (2020). Moxibustion as an adjunct for lower urinary tract symptoms associated with benign prostate enlargement: a randomized controlled pilot trial. Medicine, 99:4. https://www.ncbi.nlm.nih.gov/pmc/articles/PMC7004691/

Lokant M, \& Naz, R. (2015). Presence of PSA autoantibodies in men with prostate abnormalities (prostate cancer/benign prostatic hyperplasia/prostatitis). Andrologia; 47: 328- 332. https://pubmed.ncbi.nlm.nih.gov/24620795/

Marchand P, \& Ratinaud P. (2012). L'analyse de similitude appliqueé aux corpus textueles: les primaires socialistes pour l'election présidentielle française. Em: Actes des 11eme Journées internationales d'Analyse statistique des Données Textuelles. JADT 2012. (687-699).

Oxford Centre for Evidence-Based Medicine. (2009). Levels of evidence [Internet]. Recuperado em:http://www.cebm.net/oxfordcentre-evidence-basedmedicine-levels- evidencemarch-2009.

Park, J. S. et al. (2019). Impact of metabolic syndrome-related factors on the development of benign prostatic hyperplasia and lower urinary tract symptoms in Asian population. Medicine; 98:42. https://www.ncbi.nlm.nih.gov/pmc/articles/PMC6824778/

Parsons, J. K., et al. (2013). Obesity and benign prostatic hyperplasia: clinical connections, emerging etiological paradigms and future directions. The Journal of Urology, 189, S102-S106. https://pubmed.ncbi.nlm.nih.gov/23234610/ 
Research, Society and Development, v. 11, n. 1, e17511118310, 2022

(CC BY 4.0) | ISSN 2525-3409 | DOI: http://dx.doi.org/10.33448/rsd-v11i1.18310

Rył, A et al. (2015). "Metabolic syndrome and benign prostatic hyperplasia: association or coincidence?." Diabetology \& metabolic syndrome 7(94). https://www.ncbi.nlm.nih.gov/pmc/articles/PMC4625953/.

Santos C. M. B., et al. (2018). Sistematização da assistência de enfermagem a pessoa idosa portadora de hiperplasia prostática benigna: relato de experiência. In: Congresso Internacional de Envelhecimento Humano, 5, 2017, Maceió. Anais. Alagoas, Editora realize.

Shih, H. J. et.al. (2017). Hyperlipidemia is associated with an increased risk of clinical benign prostatic hyperplasia. The Prostate. 1-8. https://www.ncbi.nlm.nih.gov/pubmed/29119583.

Shore N. (2015). A Review of the Prostatic Urethral Lift for Lower Urinary Tract Symptoms: Symptom Relief, Flow Improvement, and Preservation of Sexual Function in Men With Benign Prostatic Hyperplasia. Current bladder dysfunction reports. 10(2): 186-192. https://www.ncbi.nlm.nih.gov/pmc/articles/PMC4424373/

Teixeira, I. D. Sanches, A. M. \& Falcão, G. (2017). Um caso clínico de sintomas Urinários baixos. Ver Port Med Geral Fam; 33:134-40. https://www.rpmgf.pt/ojs/index.php/rpmgf/article/view/12041

Wroclawski M. L, Carneiro A, Tristão R. A., Sakuramoto P. K.., Youssef J D., Lopes Neto A. C., Santiago L. H., \& Pompeo A. C. (2016). Giant prostatic hyperplasia: report of a previously asymptomatic man presenting with gross hematuria and hypovolemic shock. Einstein.;1-3. https://www.ncbi.nlm.nih.gov/pmc/articles/PMC4943789/. 